\title{
Heavy quark production from Color Glass Condensate at RHIC
}

\author{
K.L. Tuchin \\ Physics Department, Brookhaven National Laboratory, \\ Upton, NY 11973-5000, USA \\ E-mail: tuchin@quark.phy.bnl.gov
}

$\mathrm{BNL}-\mathrm{NT}-04 / 6$

\begin{abstract}
I consider production of heavy quarks in $\mathrm{pA}$ and AA collisions in the framework of the Color Glass Condensate. I discuss the heavy quark production in $\mathrm{pA}$ collisions in a classical approximation which takes into account all multiple rescatterings of a proton in a nucleus. For peripheral collisions, heavy quark production cross section can be written in $k_{T}$-factorized form. The $k_{T}$ factorization is used to construct a simple model which takes into account both classical and quantum effects in pA and AA collisions. I review the main result of calculation based on that model: open charm in the central rapidity region at RHIC gets suppressed as a function of rapidity. Although the numbers obtained for the suppression factor are model dependent, the very fact of suppression is the general feature of the Color Glass Condensate at RHIC kinematical region. It indicates the onset of quantum evolution effects in a nucleus and is universal for all inclusive processes.
\end{abstract}

The process of heavy quark production in pA collisions at high energies has three separated in time stages in the nucleus rest frame. Emission of a gluon $g$ by a proton's valence quark $q_{v}$ takes much longer time $\tau_{q_{v} \rightarrow q_{v} g}$ than a subsequent emission of a $q \bar{q}$ pair by a gluon $t_{g \rightarrow q \bar{q}} \ll \tau_{q_{v} \rightarrow q_{v}} g$. In turn, the time of interaction of a $q_{v} g q \bar{q}$ system with a nucleus is of the order of nuclear length $R_{A}$ and is negligible as compared to the evolution time of the proton wave function $\tau_{q_{v} \rightarrow q_{v} g} \gg t_{g \rightarrow q \bar{q}} \gg R_{A}$. For example, the gluon emission takes time

$$
\tau_{q_{v} \rightarrow q g} \approx \frac{2 q^{+}}{\underline{q}^{2}}=\frac{1}{x M_{N}} \gg R_{A} \simeq \tau_{\mathrm{int}},
$$

where the proton is moving in + light-cone direction. The same argument applies to the successive emission of a quark-antiquark pair by a gluon in a proton's wave function. The separation of times is ensured by strong ordering $q^{+} \gg k^{+} \gg k_{T}$, where $k^{+}$and $k_{T}$ are the quark energy and transverse momentum respectively. Therefore, processes in which gluon or heavy quarks are produced in course of the rescatterings in a nucleus are suppressed by powers of energy $p^{+}[1$.

As the result, the differential cross section for the quark production can be written in a factorized form as a convolution of the valence quark and gluon wave functions with the rescattering factor. The final results takes form [2, 3, 4]

$$
\frac{d \sigma}{d^{2} k d y}=\int d^{2} b \int d^{2} x_{0} \int d \alpha \int \frac{d^{2} x d^{2} y}{(2 \pi)^{3}} \Phi_{\mathrm{g} \rightarrow \mathrm{q} \overline{\mathrm{q}}}\left(\underline{x}, \underline{x}_{0}, \underline{y}, \alpha\right) \Phi_{\mathrm{q}_{\mathrm{v}} \rightarrow \mathrm{q}_{\mathrm{v}} \overline{\mathrm{g}}}\left(\underline{x}, \underline{x}_{0}, \underline{y}, \alpha\right)
$$


$\times e^{-i \underline{i} \cdot(\underline{x}-\underline{y})}\left(1+e^{-\frac{1}{4} \frac{C_{F}}{N_{c}}(\underline{x}-\underline{y})^{2} Q_{s}^{2}}-e^{-\frac{1}{4} \frac{C_{F}}{N_{c}}\left(\underline{x}-\underline{x}_{0}\right)^{2} Q_{s}^{2}}-e^{-\frac{1}{4} \frac{C_{F}}{N_{c}}\left(\underline{y}-\underline{x}_{0}\right)^{2} Q_{s}^{2}}\right)$,

where I assumed for simplicity that the dominant contribution comes from interaction of the $q \bar{q}$ pair with the target, while rescatterings of the gluon and the valence quark are neglected. However in general, they must be taken into account as well. In Eq. (2) I used the following notations:

$\Phi_{\mathrm{q}_{\mathrm{v}} \rightarrow \mathrm{q}_{\mathrm{v}} \overline{\mathrm{g}}}\left(\underline{x}, \underline{x}_{0}, \underline{y}, \alpha\right)=\frac{\alpha_{s} C_{F}}{\pi^{2}} \frac{\left(\alpha \underline{x}+(1-\alpha) \underline{x}_{0}\right) \cdot\left(\alpha \underline{y}+(1-\alpha) \underline{x}_{0}\right)}{\left(\alpha \underline{x}+(1-\alpha) \underline{x}_{0}\right)^{2}\left(\alpha \underline{y}+\left(1-\alpha \underline{x}_{0}\right)^{2}\right.}$,

$\Phi_{g \rightarrow q \bar{q}}\left(\underline{z}, \underline{x}, \underline{x}_{0}, \alpha\right)=\frac{\alpha_{s}}{\pi} m^{2}\left(\frac{\left(\underline{x}-\underline{x}_{0}\right) \cdot\left(\underline{y}-\underline{x}_{0}\right)}{\left|\underline{x}-\underline{x}_{0}\right|\left|\underline{y}-\underline{x}_{0}\right|} K_{1}\left(\left|\underline{x}-\underline{x}_{0}\right| m\right) K_{1}\left(\left|\underline{y}-\underline{x}_{0}\right| m\right)\right.$

$\left.\times\left[\alpha^{2}+(1-\alpha)^{2}\right]+K_{0}\left(\left|\underline{x}-\underline{x}_{0}\right| m\right) K_{0}\left(\left|\underline{y}-\underline{x}_{0}\right| m\right)\right)$,

where $Q_{s}$ is the saturation scale, $m$ is a quark mass and $\alpha=k^{+} / q^{+}$.

In the nucleus light-cone frame Eq. (2) can be interpreted as a cross section for proton scattering off the $q \bar{q}$ fluctuation of the strong nuclear gluonic field (Color Glass Condensate). At the classical level, this field is of the order $F \sim Q_{s}^{2} / g$. It is well known in QED that strong electric field $E$ can produce quark-antiquark pairs from vacuum if change of its energy $e E$ along distance $\hbar / m c$ is larger than $m c^{2}$ i. e. $e E>m^{2}$. Similarly, strong chromoelectric field can produce pairs from vacuum if $Q_{s}^{2}>m^{2}$ [5]. Theory of Color Glass Condensate predicts dependence of the saturation scale on energy and atomic number: $Q_{s} \propto A^{1 / 6} s^{\alpha_{s}} \underline{\underline{6}}$. Thus, one expects that the production pattern of heavy quarks is the same as light ones, once the collision energy is high enough.

Unfortunately, there is no calculation of the heavy quark production cross section for the AA reaction. Therefore, to get a numerical results which can be compared with RHIC experiments one uses phenomenological models which take into account the important features of the heavy quark production by strong color fields. This can be done by expanding exponents in Eq. (2) (together with terms describing gluon rescattering omitted here) to the leading order in $Q_{s}^{2}$ and Fourier-transforming to the momentum space. This way one gets the cross section for the quark production in pp collisions in $k_{T}$-factorized form [7]

$\frac{d \sigma}{d^{2} p d y_{1} d y_{2}}=\int d^{2} q_{1} \int d^{2} q_{2} \phi\left(x_{1}, \underline{q}_{1}\right) \mathcal{A}\left(\hat{s}, \hat{t}, \hat{u}, \underline{q}_{1}^{2}, \underline{q}_{2}^{2}\right) \phi\left(x_{2}, \underline{q}_{2}\right)$,

where $\mathcal{A}$ is the amplitude for the subprocess process $g^{*}\left(\underline{q}_{1}\right) g^{*}\left(\underline{q}_{2}\right) \rightarrow g g q \bar{q}$. It reduces to the familiar perturbative amplitude for on-shell gluons $g g \rightarrow q \bar{q}$ after taking the limit $\underline{q}_{1}^{2}, \underline{q}_{2}^{2} \rightarrow 0$ and averaging over azimuthal angles of $\underline{q}_{1}$ and $\underline{q}_{2}$. However, this approximation turns out to be not valid at high energies since it disagrees with the experimental data on charm and bottom production at Fermilab. $\phi(x, \underline{q})$ in (5) is the unintegrated gluon distributions such that $\phi(x, \underline{q})=d x G(x, \underline{q}) / d \underline{q}^{2}$. In principle $\phi(x, \underline{q})$ can be found from the QCD evolution equations at high energy [ 8 . However, finding the numerical solution to those equations is still not completely solved problem even in the mean-field approximation. We rather prefer to use a simple model for the unintegrated gluon distributions based on available theoretical information about the solution to the evolution equations. Assume that the cross section for the quark 

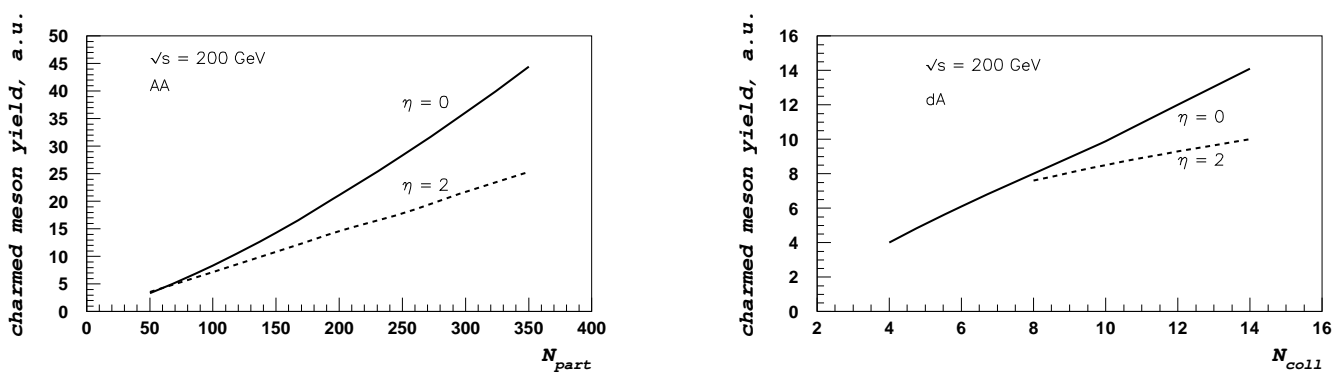

Figure 1. Dependence of the charmed meson yield on centrality at central and forward rapidities for $\mathrm{dAu}$ and $\mathrm{AuAu}$ collisions.

production in $\mathrm{pA}$ and $\mathrm{AA}$ reactions can be written in the same $k_{T}$ factorized form as for pp case (5). Then, $\phi(x, \underline{q})$ must satisfy the following conditions:

$$
\begin{aligned}
& \phi\left(x, \underline{q}^{2}\right)=\frac{4 C_{F} \pi R_{A}^{2}}{\alpha_{s} 2(2 \pi)^{2}} \ln \left(Q_{s}^{2} / \underline{q}^{2}\right), \quad \underline{q}^{2}<Q_{s}^{2}, \text { saturation } \\
& \phi\left(x, \underline{q}^{2}\right) \sim \underline{q}^{-2}, \quad q^{2}>k_{\text {geom }}^{2}, \text { pQCD behavior }
\end{aligned}
$$

where $k_{\text {geom }}^{2} \gg Q_{s}^{2}$ is the "extended geometric scaling" [9] momentum squared, and

$$
\phi\left(x, \underline{q}^{2}\right)=\phi\left(\underline{q}^{2} / Q_{s}^{2}\right), \quad \underline{q}^{2}<k_{\text {geom }}^{2}, \text { "extended geometric scaling" (8) }
$$

Of course there is more than one function satisfying these conditions. In my paper with D. Kharzeev [5] a parametrization is suggested which is inspired by the double logarithmic approximation to the DGLAP and BFKL equations. Our numerical calculations have been done with that function. The results of calculations are presented in Fig. 1] and Fig. 2 ]

The charmed meson yield shown in Fig. 1 gets suppressed as one goes from central rapidity $y=0$ to forward rapidity $\eta=2$ both in $\mathrm{pA}$ and AA collisions. This is because the saturation scale of a nucleus increases with rapidity. At RHIC $Q_{s}^{2} \simeq 2 e^{0.3 y} \mathrm{GeV}^{2}$. Therefore, at midrapidity $Q_{s}^{2}<m_{c}^{2}$ and the charmed quarks are produced perturbatively. As the result yield scales like

$$
\left.\frac{d N_{A A}}{d \eta}\right|_{\eta=0} \sim A^{4 / 3} \sim N_{\text {coll }} .
$$

At forward rapidity $Q_{s}^{2}>m_{c}^{2}$ implying that

$$
\left.\frac{d N_{A A}}{d \eta}\right|_{\eta \geq 2} \sim A \sim N_{\text {part }} .
$$

The same formulas hold for dA after replacement of $N_{\text {part }}$ by $N_{\text {part }}^{A u}$ and $N_{\text {coll }}$ by $N_{\text {coll }}^{A u}$.

The suppression effect is insensitive to high energy quantum evolution effects which change the anomalous dimension of the gluon distribution functions for transverse momenta satisfying $Q_{s}^{2}<\underline{q}^{2}<k_{\text {geom }}^{2}$. If however one applies an infrared cutoff on a spectrum at transverse momenta of the order of $Q_{s}$, then the region $\underline{q}^{2}<k_{\text {geom }}^{2}$ where the quantum effects are important will dominate the yield. The resulting nuclear modification factors scale like $R_{A A} \sim 1 / A^{1 / 3} \sim 1 / N_{\text {part }}^{1 / 3} \simeq 0.5$ and 


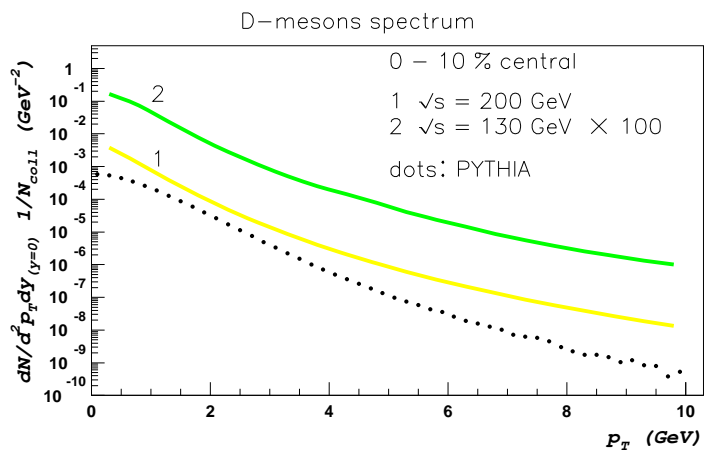

Figure 2. $\frac{1}{2}\left(D^{0}+\bar{D}^{0}\right)$ spectra in heavy-ion collisions at $\sqrt{s}=130 \mathrm{GeV}$ and $\sqrt{s}=200 \mathrm{GeV}$.

$R_{d A} \sim 1 / A^{1 / 6} \sim 1 / \sqrt{N_{\text {part }}^{A u}} \simeq 0.75$ in the forward region. As we have already noted, these suppression factors at forward rapidities are of the same order of magnitude as in inclusive meson production case, since at low- $x$ (forward rapidities) production pattern of charmed quarks is the same as the light ones.

In Fig. 2] we compare the open charm spectrum obtained from our saturation model based on $k_{T}$-factorization with predictions based on collinear factorization approach (PYTHIA). We observe that PYTHIA spectrum is significantly softer than ours. Therefore, we predict much harder open charm spectrum. Of course, the steepness of the PYTHIA spectrum depends on the intrinsic momentum parameter $k_{0}$. Our spectrum can probably be reproduced by PYTHIA if one takes $k_{0} \simeq Q_{s} \gg \Lambda_{\mathrm{QCD}}$ and fixes the $K$-factor. This large value of intrinsic $k_{T}$ would however signal the breakdown of the collinear factorization approach.

\section{Acknowledgments}

The author is indebted to Dmitri Kharzeev for collaborating in most of the results discussed in this publication. This research was supported by the U.S. Department of Energy under Grant No. DE-AC02-98CH10886.

\section{References}

[1] V. N. Gribov, B. L. Ioffe and I. Y. Pomeranchuk, Sov. J. Nucl. Phys. 2, 549 (1966) [Yad. Fiz. 2, 768 (1965)];

[2] B. Z. Kopeliovich and A. V. Tarasov, Nucl. Phys. A 710, 180 (2002) arXiv:hep-ph/0205151.

[3] K. Tuchin, arXiv:hep-ph/0401022

[4] J.P. Blaizot, F. Gelis, R. Venugopalan, arXiv:hep-ph/hep-ph/0402257

[5] D. Kharzeev and K. Tuchin, arXiv:hep-ph/0310358

[6] E. Levin and K. Tuchin, Nucl. Phys. B 573, 833 (2000) arXiv:hep-ph/9908317, Nucl. Phys. A 693, 787 (2001) arXiv:hep-ph/0101275.

[7] E. M. Levin, M. G. Ryskin, Y. M. Shabelski and A. G. Shuvaev, Sov. J. Nucl. Phys. 53, 657 (1991) [Yad. Fiz. 53, 1059 (1991)]; S. Catani, M. Ciafaloni and F. Hautmann, Nucl. Phys. B 366, 135 (1991); J. C. Collins and R. K. Ellis, Nucl. Phys. B 360, 3 (1991); F. Gelis and R. Venugopalan, arXiv:hep-ph/0310090 
[8] I. Balitsky, Nucl. Phys. B 463, 99 (1996) arXiv:hep-ph/9509348; Y. V. Kovchegov, Phys. Rev. D 60, 034008 (1999) arXiv:hep-ph/9901281 . A. Kovner, J. G. Milhano and H. Weigert, Phys. Rev. D 62, 114005 (2000) arXiv:hep-ph/0004014. E. Iancu and L. D. McLerran, Phys. Lett. B 510, 145 (2001) arXiv:hep-ph/0103032.

[9] E. Iancu, K. Itakura and L. McLerran, Nucl. Phys. A 708, 327 (2002) arXiv:hep-ph/0203137, E. Levin and K. Tuchin, Nucl. Phys. A 691, 779 (2001) arXiv:hep-ph/0012167, 